\title{
Isorhamnetin suppresses colon cancer cell growth through the PI3K-Akt-mTOR pathway
}

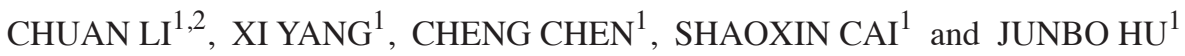 \\ ${ }^{1}$ Department of Gastrointestinal Surgery, Tongji Hospital, Huazhong University of Science and Technology, \\ Wuhan, Hubei 430030; ${ }^{2}$ General Surgery Center of PLA, Southwest Hospital, \\ The Third Military Medical University, Chongqing 400038, P.R. China
}

Received March 16, 2013; Accepted August 30, 2013

DOI: $10.3892 / \mathrm{mmr} .2014 .1886$

\begin{abstract}
Isorhamnetin, a flavonoid isolated from the fruits of herbal medicinal plants, such as Hippophae rhamnoides L., exerts anticancer effects similar to other flavonoids. However, the effect of isorhamnetin on colorectal cancer (CRC) and the underlying molecular mechanism are unclear. This study aimed to determine the effect of isorhamnetin on the proliferation of cells from the human CRC cell lines, HT-29, HCT116 and SW480. It was demonstrated that isorhamnetin suppressed the proliferation of cells from all three cell lines, induced cell cycle arrest at the G2/M phase and suppressed cell proliferation by inhibiting the PI3K-Akt-mTOR pathway. Isorhamnetin also reduced the phosphorylation levels of Akt (ser473), phosph-p70S6 kinase and phosph-4E-BP1 (t37/46) protein, and enhanced the expression of Cyclin B1 protein. Therefore, this compound was revealed to be a selective PI3K-Akt-mTOR pathway inhibitor, and may be a potent anticancer agent for the treatment of CRC, as it restrains the proliferation of CRC cells.
\end{abstract}

\section{Introduction}

Flavonoids are widely distributed in plants and have numerous functions, such as antioxidant activity in vitro (1) and potential anticancer activity (2). Isorhamnetin (3'-methoxy-3,4',5,7-tetrahydroxyflavone; Fig. 1A) is a flavonoid extracted from plants such as Persicaria thunbergii H. and Hippophae rhamnoides L. This compound is used to treat cardiovascular diseases and hemorrhage due to its antioxidative and metabolic effects $(3,4)$. Its anticancer effects have also been reported (5-9); however, the mechanisms underlying these effects remain unclear.

Correspondence to: Professor Junbo $\mathrm{Hu}$, Department of Gastrointestinal Surgery, Tongji Hospital, Huazhong University of Science and Technology, 1095 Jiefang Road, Qiaokou, Wuhan, Hubei 430030, P.R. China

E-mail: sanrixq@live.com

Key words: isorhamnetin, PI3K-Akt-mTOR, inhibitor, colon cancer
Colon cancer accounts for $\sim 10 \%$ of all tumors and is the most common type of cancer worldwide (10). One of the most common genetic factors of this cancer is a PI3K mutation (11). The PIK3CA gene is mutated in $\sim 20 \%$ of colorectal cancers (CRCs), which activates the PI3K-Akt-mTOR signal pathway (12). This pathway significantly affects cell proliferation, metabolism and the stress response, making it an important target in the treatment of CRC.

We found that the Akt activity of colon cancer cells can be inhibited by isorhamnetin in our pre-experiment. Thus, it was hypothesized that isorhamnetin inhibits CRC by suppressing the PI3K-Akt-mTOR pathway. Accordingly, the present study was performed to validate this hypothesis.

\section{Materials and methods}

Reagents. Isorhamnetin was purchased from Chromadex (Irvine, CA, USA), dissolved in dimethylsulfoxide (DMSO) and diluted to $20 \mathrm{mmol} / \mathrm{l}$. Rapamycin and LY294002 were purchased from Cell Signaling Technology, Inc. (Beverly, MA, USA). An MTT kit was purchased from Promoter (Wuhan, Hubei, China). Propidium iodide (PI) was obtained from Sigma-Aldrich (St. Louis, MO, USA). The BCA Protein Assay kit, ECL detection system and horseradish peroxidaseconjugated secondary antibody were obtained from Pierce (Rockford, IL, USA). Antibodies against AKT, phosphorylated (p)-AKT (ser473) and 4E-PB1 were obtained from Cell Signaling Technology, Inc.. Antibodies against p70S6k and p-p70S6K were purchased from Epitomics, Inc. (Burlingame, CA, USA); antibodies against p21 and Cyclin B1 were obtained from BD Biosciences (San Jose, CA, USA); and an antibody against glyceraldehyde 3-phosphate dehydrogenase (GAPDH) was purchased from Santa Cruz Biotechnology, Inc. (Santa Cruz, CA, USA).

Cell lines and culture. SW480, HCT116 and HT-29 cell lines were purchased from the Cell Bank of Type Culture Collection of the Chinese Academy of Sciences (Shanghai, China). SW480 and HCT116 cells were cultured in Dulbecco's modified Eagle's medium/high-glucose medium with $10 \%$ fetal bovine serum (FBS; Hyclone, Waltham, MA, USA) in a humidified atmosphere $\left(37^{\circ} \mathrm{C}, 5 \% \mathrm{CO}_{2}\right)$. HT-29 cells were cultured in $10 \% \mathrm{FBS} / \mathrm{McCoy}$ 's $5 \mathrm{a}$ under the same conditions. 
For all experiments, the cells were grown to $90 \%$ confluency and harvested every 2-3 days.

MTT assay. An MTT assay was performed as described previously by Mosmann (13). MTT was dissolved in DMSO to $5 \mathrm{mg} /$ $\mathrm{ml}$. The cells were digested, counted and seeded onto 96-well culture plates at a density of $5 \times 10^{3}$ cells per well. Subsequently, the cells were incubated overnight and the culture medium was replaced with isorhamnetin at concentrations of $0,10,20$, 40 and $80 \mu \mathrm{mol} / \mathrm{l}$. After 3 days, $10 \mu \mathrm{l}$ MTT $(5 \mathrm{mg} / \mathrm{ml})$ was added to each well and cells were cultured for another $4 \mathrm{~h}$. The medium was then discarded and $150 \mu \mathrm{l}$ DMSO was added to the culture wells. After gently agitating for $10 \mathrm{~min}$ with a table shaker (Premiere, Changzhou, China) at $40 \mathrm{rpm}$ for $10 \mathrm{~min}$, the samples were placed on a microplate reader (Biotek, Winooski, VT, USA) and the absorbance was detected at $550 \mathrm{~nm}$. Data were calculated and the growth inhibition curve was constructed.

Western blot analysis. Cells were seeded onto 6-well plates following treatment with isorhamnetin $(0,20$ and $40 \mu \mathrm{mol} / \mathrm{l})$, and proteins were harvested and collected by NP-40 lysis buffer (Beyotime, Haimen, China). The protein concentrations were determined by a Bicinchoninic Acid Protein Assay kit. Subsequently, $40 \mu \mathrm{g}$ of each protein sample was added to $12 \%$ sodium dodecyl sulfate-polyacrylamide gel electrophoresis gel. Following electrophoresis at $100 \mathrm{~V}$ for $2 \mathrm{~h}$, the proteins were transferred to polyvinylidene difluoride membranes at $350 \mathrm{~mA}$ for $90 \mathrm{~min}$. The membranes were then blocked with 5\% non-fat milk in Tris-buffered saline and Tween 20 (TBST), incubated with primary antibody at $4^{\circ} \mathrm{C}$ overnight, washed three times with TBST, incubated for $1 \mathrm{~h}$ at room temperature and washed again three times. The chemical signal was detected using an enhanced chemiluminescence detection system. The chemical detection instrument used was FluorChem FC2 (Cell Biosciences, Murrieta, CA, USA).

PI staining. Cells were seeded onto 6-well plates, treated with isorhamnetin $(0,20$ and $40 \mu \mathrm{mol} / \mathrm{l})$ for $24 \mathrm{~h}$ and harvested at the exponential phase. The samples were fixed with $75 \%$ ice-cold ethyl alcohol overnight at $-20^{\circ} \mathrm{C}$. On the following day, the cells were centrifuged at $300 \mathrm{x}$ g for 5 min (Beckman, Brea, CA, USA) and added drop wise with ethyl alcohol. The samples were washed three times with phosphate buffered saline (PBS), resuspended in $100 \mu 1 \mathrm{PBS}$, combined with $10 \mu \mathrm{l}$ of $500 \mu \mathrm{g} / \mathrm{ml}$ PI and $5 \mu \mathrm{l}$ of $10 \mathrm{mg} / \mathrm{ml}$ RNase A, and then incubated for $2 \mathrm{~h}$ in the dark. Following this, $500 \mu \mathrm{l}$ PBS was added prior to flow cytometry (FCM) analysis.

Bromodeoxyuridine (BrdU) corporation. Approximately $300 \mathrm{ng} / \mathrm{ml}$ BrdU (Sigma-Aldrich) was added to the medium, which was then incubated for $30 \mathrm{~min}$ prior to harvesting. Cells were collected and fixed with $75 \%$ ice-cold ethyl alcohol overnight at $-20^{\circ} \mathrm{C}$. On the following day, the samples were washed once and treated with $0.5 \mathrm{ml} 2 \mathrm{M} \mathrm{HCl}$ for $40 \mathrm{~min}$. Subsequently, $0.5 \mathrm{ml} 0.1 \mathrm{M}$ sodium borate ( $\mathrm{pH} 8.5$ ) was added for neutralization, and the samples were incubated with anti-BrdU for $1 \mathrm{~h}$ and a secondary antibody for $30 \mathrm{~min}$. After washing three times, PI and RNase were added to the samples for another $30 \mathrm{~min}$ of incubation. Finally, all samples were analyzed by FCM.
A<smiles>COC1=C(O)CCC(c2oc3cc(O)cc(O)c3c(=O)c2O)=C1</smiles>

B MTT assay

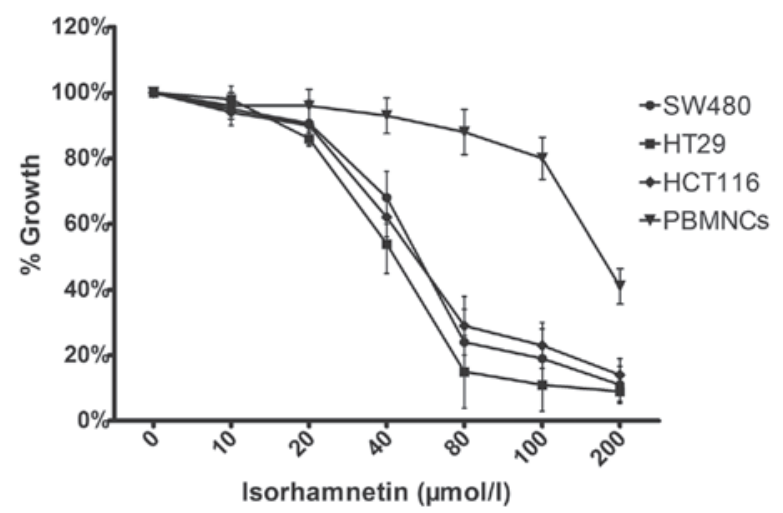

C

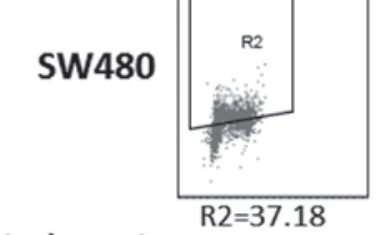

Isorhamnetin $(\mu \mathrm{mol} / \mathrm{ml})$ :

0
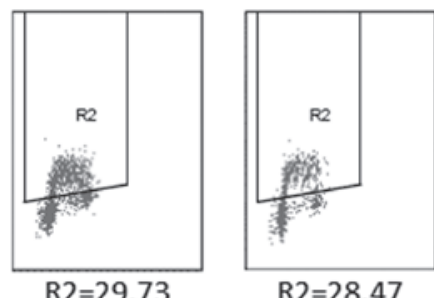

20

40

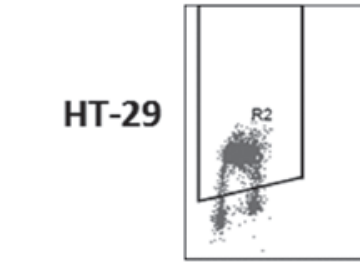

Isorhamnetin

$\mathrm{R} 2=71.4$

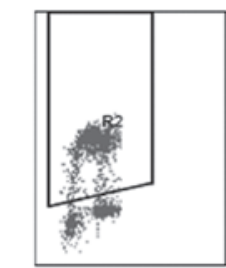

$\mathrm{R} 2=66.59$

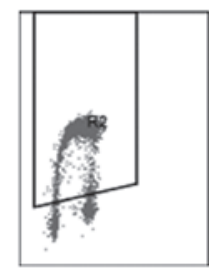

$R 2=58.94$ $(\mu \mathrm{mol} / \mathrm{ml})$ :

0

20

40

D Quantitative statistical analysis of BrdU

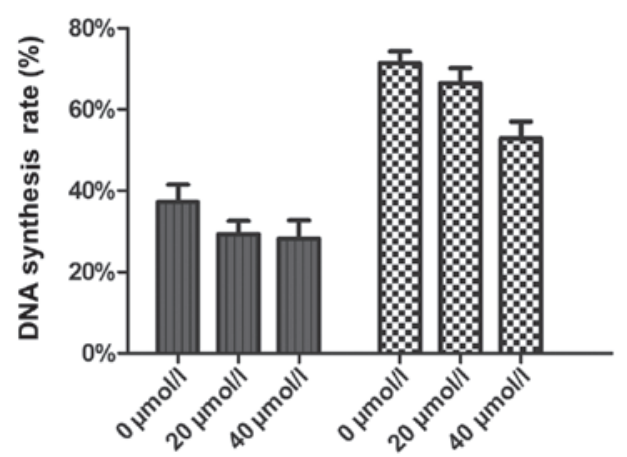

Figure 1. Isorhamnetin inhibits the proliferation of colon cancer. (A) Chemical structure of isorhamnetin. (B) Curve of inhibition of CRCs and PBMNCs against isorhamnetin (MTT assay). (C) BrdU assay of two CRC lines. (D) Quantitative statistical analysis results of the BrdU assay. PBMNCs, peripheral blood mononuclear cells; CRCs, colorectal cancers; BrdU; bromodeoxyuridine 

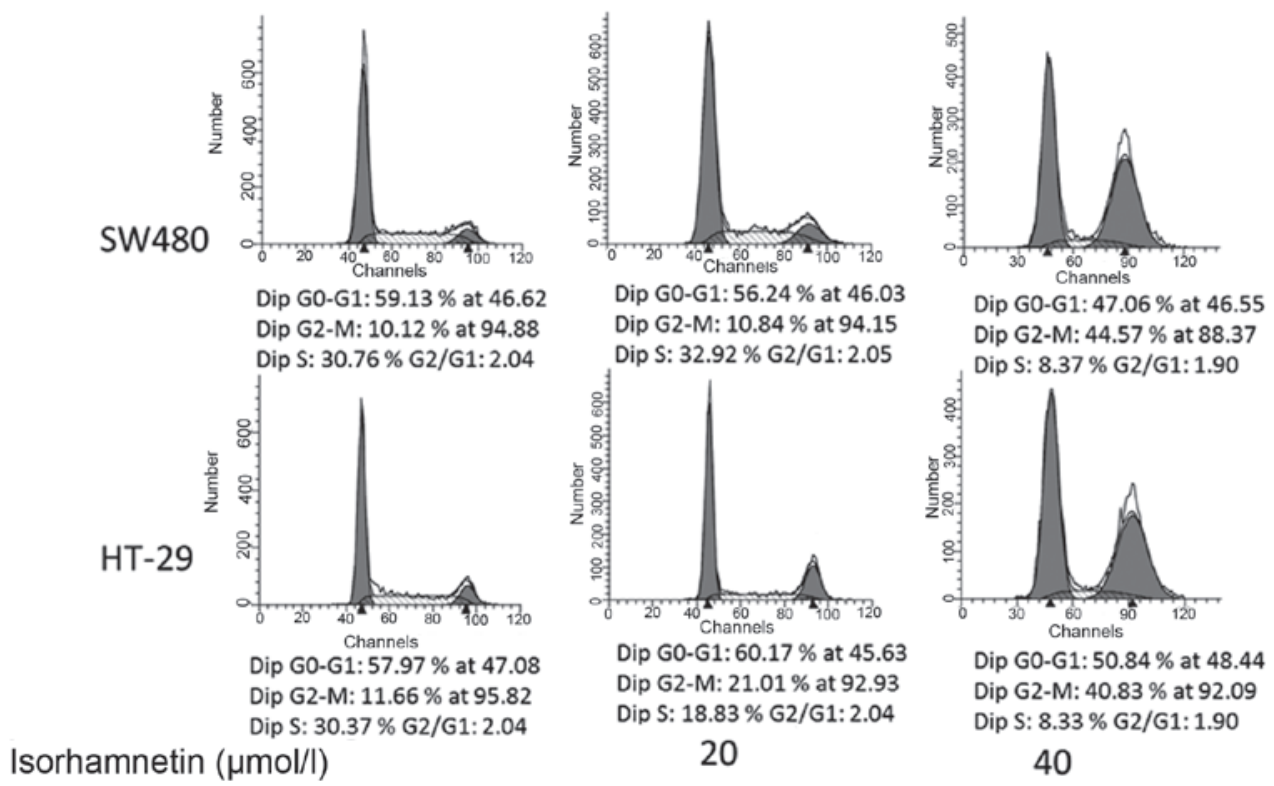

Figure 2. Isorhamnetin induces cell cycle arrest at the G2/M phase. SW480 and HT-29 cells were treated for $24 \mathrm{~h}$ with isorhamnetin at three concentrations $(0,20$ and $40 \mu \mathrm{mol} / \mathrm{l})$ and the cell cycle stage was detected by flow cytometry analysis.

Statistical analysis. All statistical analyses were conducted using SPSS, version 12.0 (SPSS Inc., Chicago, IL, USA). The values are presented as the mean \pm SD. Student's t-test was used to determine the statistical significance of the differences between the treatment group and the control group. The data were statistically analyzed by analysis of variance and $\mathrm{P}<0.05$ was considered to indicate a statistically significant difference.

\section{Results}

Isorhamnetin suppresses the proliferation of CRC cells. The effect of isorhamnetin on the proliferation of CRC cells was determined by an MTT assay. The three cell lines were treated with isorhamnetin at five concentrations $(0,10,20,40$ and $80 \mu \mathrm{mol} / \mathrm{l}$ ) for three days. Peripheral blood mononuclear cells (PBMNCs) were added to verify the cytotoxicity of isorhamnetin. The results shown in Fig. 1B indicated that the inhibition effect was dose dependent. The $\mathrm{IC}_{50}$ values of isorhamnetin were as follows: $56.24 \pm 1.25 \mu \mathrm{mol} / 1$ for SW480 cells, $54.87 \pm 2.13 \mu \mathrm{mol} / 1$ for HCT116 cells and 43.85 $\pm 3.45 \mu \mathrm{mol} / 1$ for HT-29 cells. The $\mathrm{IC}_{50}$ value of isorhamnetin in PBMNCs was $170 \mu \mathrm{mol} / 1$. This result showed that the $\mathrm{IC}_{50}$ value of isorhamnetin in PBMNCs was considerably higher than that of the three CRC cell types. Thus, it is possible to eliminate the cytotoxicity of isorhamnetin to non-tumor cells. The effect of isorhamnetin on HCT116 cells has previously been demonstrated (8), therefore the remaining two cell lines were selected for subsequent experiments.

A BrdU assay was directly used to detect cell proliferation, as shown in Fig. 1C and D. The results were similar to those of the MTT assay. At $40 \mu \mathrm{mol} / \mathrm{l}$ isorhamnetin, the average inhibition ratio was $10 \pm 4 \%(\mathrm{P}=0.002)$ in the $\mathrm{SW} 480$ cell line and $12 \pm 3.6 \%(\mathrm{P}=0.001)$ in the HT-29 cell line.

Isorhamnetin induces G2/M growth arrest in human CRC cells. PI staining was conducted to further investigate whether isorhamnetin affected the cell cycle. Following treatment with isorhamnatin at three concentrations $(0,20$ and $40 \mu \mathrm{mol} / \mathrm{l})$, the average proportions of the $\mathrm{G} 2 / \mathrm{M}$ phase in each group were respectively as follows (Fig. 2): 11.6, 21 and 44.57\% in SW480 cells; and 10, 10.9 and $40.83 \%$ in HT-29 cells. The G2/M phase of HT-29cells increased by $9.5 \%$ at $20 \mu \mathrm{mol} / \mathrm{l}(\mathrm{P}=0.003)$ and $32.8 \%(\mathrm{P}=0.004)$ at $40 \mu \mathrm{mol} / 1$. The increase in $\mathrm{SW} 480$ cells was $30.8 \%$ at $40 \mu \mathrm{mol} / 1(\mathrm{P}=0.0067)$, but no obvious change was observed at $20 \mu \mathrm{mol} / 1(\mathrm{P}=0.65)$. The result was similar in HCT116 cells (data not shown). These data suggested that isorhamnetin is able to induce cell cycle arrest at the G2/M phase in a dose-dependent manner.

Isorhamnetin inhibits the phosphorylation of Akt in CRC cells. Similar to other flavonoids, isorhamnetin may affect the receptor-tyrosine signaling pathway. Yang et al (14) reported that the phosphorylation of Akt may be suppressed by chrysoeriol, a type of flavonoid. It was hypothesized that isorhamnetin also affects the phosphorylation level of Akt in $\mathrm{CRC}$ cells and, therefore, in the present study possible changes were detected by western blot analysis. As shown in Fig. 3, the two cell lines in the dose gradient group were treated with isorhamnetin for $24 \mathrm{~h}$ at different concentrations. The results showed that phospho-Akt (ser473) was suppressed at $20 \mu \mathrm{mol} / 1$ isorhamnetin and the change was significant at $40 \mu \mathrm{mol} / 1$ isorhamnetin. All cells in the time gradient group were treated with the same concentration $(20 \mu \mathrm{mol} / \mathrm{l})$ at six time points, 0 , $6,12,18,24$ and $36 \mathrm{~h}$. It was identified that the phosphorylation level of Akt (ser473) was suppressed after $12 \mathrm{~h}$ treatment with isorhamnetin and the change was significant after $24 \mathrm{~h}$. Thus, isorhamnetin downgraded the phosphorylation level of Akt (ser473) in a dose- and time-dependent manner.

Isorhamnetin affects the phosphorylation of p70S6K and $4 E-B P 1$ protein. Isorhamnetin degraded the phosphorylation level of $\mathrm{Akt}$; thus, on the following day it 

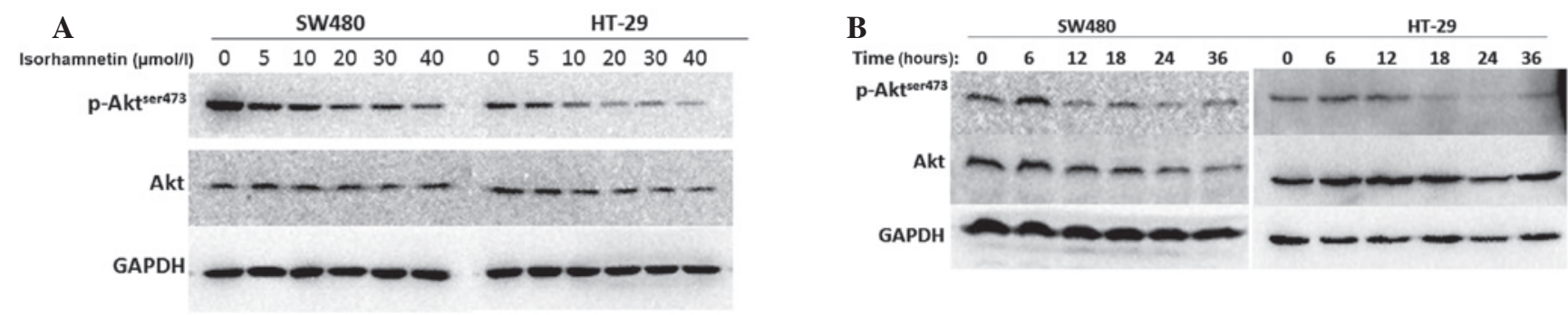

Figure 3. Isorhamnetin inhibits the phosphorylation level of Akt (ser473) in a time- and dose-dependent manner. (A) SW480 and HT-29 cells were treated with isorhamnetin at different concentrations. After $24 \mathrm{~h}$, the cells were harvested and the phosphorylation level of Akt was detected by western blot analysis. At $10 \mu \mathrm{mol} / 1$ isorhamnetin, the phosphorylation level was inhibited. (B) The two cell lines were treated with $20 \mu \mathrm{mol} / 1$ isorhamnatin for $0,6,12,18,24$ and $36 \mathrm{~h}$. The phosphorylation level of Akt decreased after $12 \mathrm{~h}$.

A

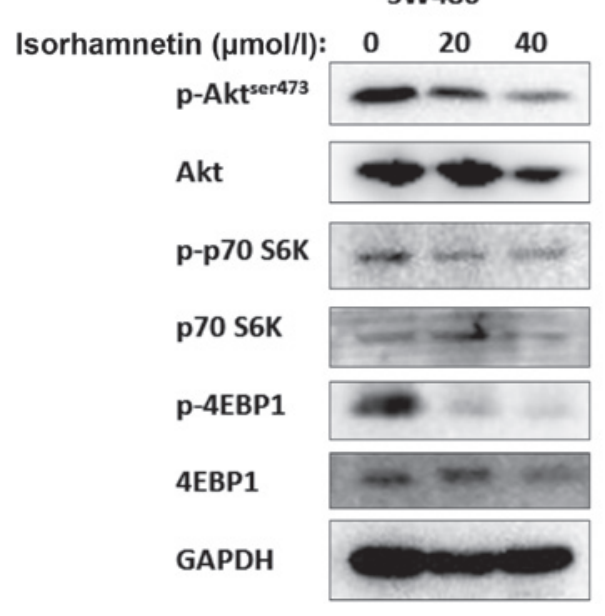

HT-29

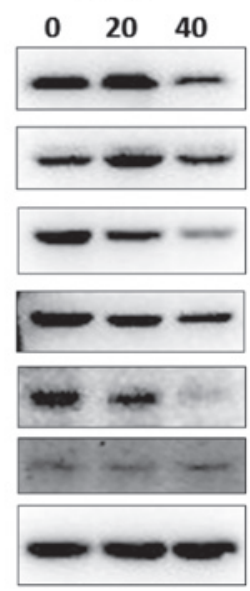

B

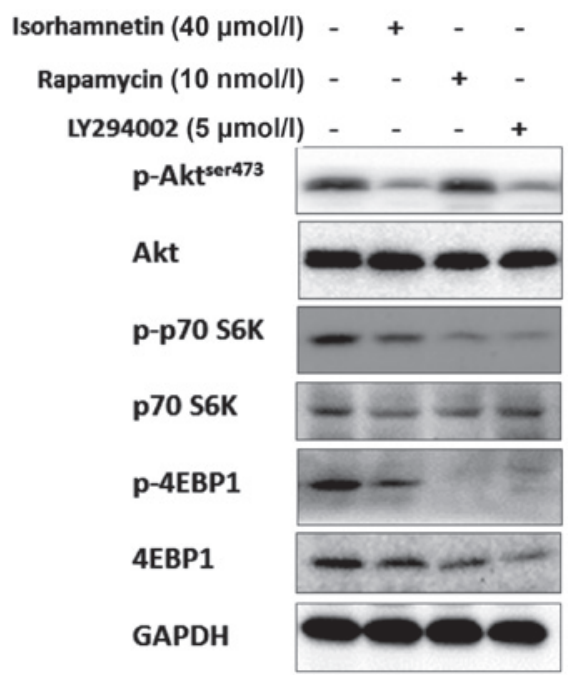

Figure 4. Effects of isorhamnetin on the Akt pathway. (A) SW480 and HT-29 cells were treated with the control medium or at the two concentrations (20 and $40 \mu \mathrm{mol}$ ) of isorhamnetin for $24 \mathrm{~h}$. Protein levels were detected by western blot analysis. The phosphorylation level of Akt, p70S6K and 4E-BP1 proteins were inhibited by isorhamnetin. (B) Different effects of isorhamnetin, LY294002 and rapamycin on the Akt pathway. SW480 cells were treated with three reagents at the indicated dose for $24 \mathrm{~h}$ in complete medium, and then lysed in lysis buffer for western blot analysis of the phosphorylation and expression of Akt, p70S6K and 4E-BP1.

was determined whether isorhamnetin affected the other Akt-related proteins, particularly those downstream of Akt. Certain PI3K-AKT-mTOR-related proteins were detected and it was identified that the phosphorylation levels of the two predominant mTOR target proteins, p70S6K and 4E-BP1, were inhibited following treatment with isorhamnetin. The results are shown in Fig. 4. In contrast to other PI3K inhibitors, isorhamnetin did not induce feedback activation of Akt, as is the case with rapamycin. The inhibition effect of isorhamnetin on p70S6K and 4E-BP1 was similar to (although marginally lower than) that of LY294002. Thus, it was inferred that isorhmantin inhibited the PI3K-Akt-mTOR pathway. Moreover, the phosphorylation levels of GSK $3 \beta$ protein were not changed (data not shown).

Isorhamnetin increases the level of Cyclin B1 proteins. To determine whether the G2/M arrest induced by isorhamnetin in CRC cells was due to the changes in cell cycle-related proteins, western blot analysis was performed to assess the expression of these proteins, including Cyclin B1, Cyclin D1 and p21. As shown in Fig. 5, the expression levels of Cyclin D1 were not changed in HT-29 cells but increased in SW480 cells; those of p21 were not changed in HT-29 cells but decreased in
SW480
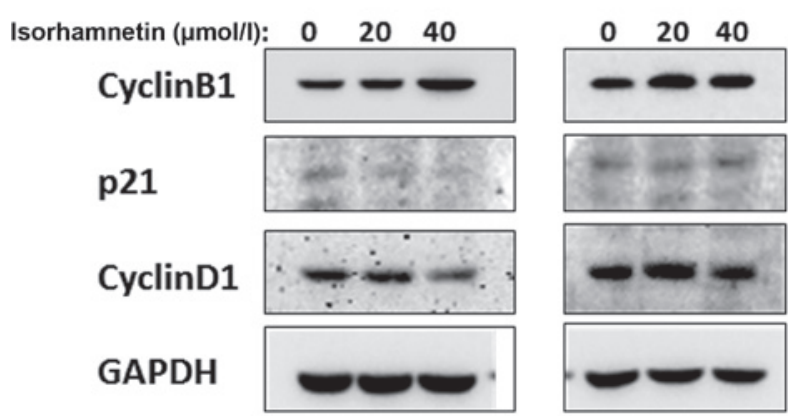

Figure 5. Effect of isorhamnetin on the expression of cell cycle-related proteins. The SW480 and HT-29 cells were treated with control medium or the two concentrations (20 and $40 \mu \mathrm{mol}$ ) of isorhamnetin for $24 \mathrm{~h}$. Protein levels were detected by western blot analysis using antibodies against Cyclin B1, Cyclin D1, p21 and GAPDH. GAPDH blotting showed equal loading. GAPDH, glyceraldehyde 3-phosphate dehydrogenase.

SW480 cells; and the expression levels of Cyclin B1 increased in the two cell types. The Cyclin B1-CDK1 complex was correlated with the G2-mitosis phase, i.e., the increase in Cyclin B1 expression levels prolonged the G2/M phase. Therefore, the 
isorhamnetin-induced cell cycle arrest was associated with the increase in Cyclin B1 expression levels. Cyclin D1 and p21 expression levels did not change similarly in the two types of cells; thus, it was not possible to confirm the effect of isorhamnetin on these two cell cycle proteins.

\section{Discussion}

The PI3K-Akt-mTOR signaling pathway is important throughout the life of a cell, including during proliferation, glucose metabolism and for survival $(15,16)$. In normal cells, this pathway benefits growth and metabolism, and its function is controlled by other regulatory proteins. One negative regulatory protein is phosphatase and tensin homolog (PTEN), which is known to be an antioncogene. The PTEN gene is usually inactivated due to mutation, deletion or epigenetic silencing. By contrast, the PI3K-Akt-mTOR pathway is frequently activated in tumorgenesis. Thus, this signaling pathway is widely recognized as a key regulator of cancer cells and is an important target of anticancer drugs $(17,18)$. Numerous small-molecule inhibitors that inhibit the aberrant PI3K-Akt-mTOR pathway have been developed $(19,20)$. However, a number of unidentified inhibitors may be toxic. Rapamycin was the first mTORC1 inhibitor approved by the Food and Drug Administration (21). The number of studies in this field are increasing, but no satisfactory inhibitor that may be used to cure cancer has been identified. The possible reasons for this include incomplete inhibition and secondary activation. Rapamycin inhibits mTORC1 but simultaneously triggers a negative feedback mechanism that activates an upstream bypass (22). This bypass persistently activates the $\mathrm{p}-\mathrm{S} 6 \mathrm{~K} 1$ protein, which is downstream of mTORC1 and leads to treatment failure.

Flavonoids are a group of natural plant secondary metabolites or yellow pigments, with a structure similar to that of flavones. They are most commonly known for their antioxidant activity in vitro. Flavonoids are able to inhibit tumor invasion, and their antiproliferative effects are associated with their structure (23). However, the potential mechanisms underlying their anticancer activity are unclear. Isorhamnetin is a dietary flavonoid found in several fruits, such as apples, pears and blackberries. It is also a predominant plasma metabolite of quercetin. A previous study has shown that quercetin induces cell cycle arrest at G0/G1 in SK-Br3 breast carcinoma cells (24). Additionally, quercetin blocks SW480 cells at the G2/M phase by suppressing Cyclin D1 and survivin expression. Similar results have been obtained after treating cells with structurally related analogs of quercetin $(25,26)$. Isorhamnetin has a structure similar to other flavonoids; it is an intermediate 3'O-methylated metabolite of quercetin. Its antiproliferative effect has been confirmed in skin cancer cells, esophageal squamous carcinoma cells and hepatocellular carcinoma cells $(6,8,9)$. Jaramillo et al (5) investigated the effect of isorhamnetin on the HCT116 cell line. In the present study, the suppressive effect was also identified in three CRC cell lines, and isorhamnetin was observed to inhibit the PI3K-Akt-mTOR pathway and affect cell cycle-related proteins.

Accordingly, the inhibition ratio of isorhmnetin was investigated by an MTT assay. The results showed that isorhamnetin suppressed the growth of the three CRC cell lines.
The $\mathrm{IC}_{50}$ value after $72 \mathrm{~h}$ was $\sim 50 \mu \mathrm{mol} / 1$ and growth was slow, as shown by the BrdU assay. Cell cycle analysis confirmed that isorhamnetin degraded the G1 phase and induced G2/M phase arrest. The findings of the present study were consistent with those of a previous study showing that isorhamnetin inhibited proliferation and induced cell cycle arrest in human HCT116 cells (27).

Previous studies have shown that isorhamnetin affected the phosphorylation level of Akt in JB6 and A431 skin cancer cells (6). Thus, western blot analysis was conducted on the cell lines in this study to detect the phosphorylation of Akt after different time periods and with different doses of isorhamnetin. The results showed that the phosphorylation of Akt was inhibited with $20 \mu \mathrm{mol} / \mathrm{l}$ isorhamnetin after $12 \mathrm{~h}$ and the effective time point and dose indicated that this compound was low-dose isorhamnetin-dependent and had a rapid onset. Akt is the key factor downstream of PI3K, to a certain extent, it could reflect whether isorhamnetin inhibited the entire downstream pathway. The predominant downstream factors of PI3K-Akt-mTOR, p70S6K and 4E-BP1 were also detected. In SW480 and HT-29 cells, isorhamnetin suppressed AKT and downregulated the phosphorylation levels of p70S6K and 4E-BP1. Thus, it was proposed that isorhamnetin may suppress the growth of CRC cells by inhibiting the PI3K-Akt-mTOR-4E-BP1/p70S6K signaling pathway. In addition, rapamycin induces the feedback activation of Akt signaling through an IGF-1R-dependent mechanism (22) and LY294002 is a synthetic PI3K inhibitor based on quercetin, which inhibits a broad range of protein kinases, but has been identified to be toxic (28). This key PI3K signaling pathway is highly activated in the majority of malignant types of cancer, and contributes to malignant transformation, proliferation and metastasis of tumor cells $(29,30)$.

The expression pattern of cell cycle-related proteins was analyzed further. The cell cycle checkpoint is a monitoring mechanism that ensures the faithful replication of cells (31). Cyclin B1 is produced at the late $\mathrm{S}$ phase and degraded at the meta-mitosis phase. The Cyclin B1-CDK1 complex aids in $\mathrm{G} 2 / \mathrm{M}$ transition, resulting in entry of the cells into mitosis. The G2/M transition phase is an important checkpoint of the cell cycle and a target for the inhibition of cell proliferation. A previous study has demonstrated that Cyclin B1 is enriched when cells are arrested at the G2/M checkpoint (32). When SW480 and HT-29 cells were treated with $40 \mu \mathrm{mol} / 1$ isorhamnetin, the expression of Cyclin B1 increased compared with that in the controls. Cyclin D1, which controls the G1/S checkpoint, did not change when the cells were treated with chrysoeriol. These results suggested that isorhamnetin predominantly induced G2/M cell cycle arrest, which may be attributed to the increase in Cyclin B1 expression levels.

All of the aforementioned biological activities were exerted in vitro by isorhamnetin at small concentrations; however, the values may not coincide with in vivo results. In conclusion, cancer is a proliferative disease characterized by an imbalance between oncogenes and antioncogenes. Cancer cells often grow rapidly, escape from the cell-cycle checkpoints and cause abnormal activation of certain signaling pathways. In the present study, isorhamnetin significantly inhibited the proliferation of CRC cells, delayed the G2/M cell cycle phase and inhibited the PI3K-Akt-mTOR pathway. 
Thus, isorhamnetin may potentially serve as an agent for CRC therapy as evidenced by preliminary data. However, additional studies are required to explain the role of isorhamnetin as a PI3K-Akt-mTOR pathway inhibitor.

\section{References}

1. Murtaza I, Adhami VM, Hafeez BB, Saleem M and Mukhtar H: Fisetin, a natural flavonoid, targets chemoresistant human pancreatic cancer AsPC-1 cells through DR3-mediated inhibition of NF- $\kappa$ B. Int J Cancer 125: 2465-2473, 2009.

2. Lin Y, Shi R, Wang X and Shen HM: Luteolin, a flavonoid with potential for cancer prevention and therapy. Curr Cancer Drug Targets 8: 634-646, 2008.

3. Devi VG, Rooban BN, Sasikala V, Sahasranamam V and Abraham A: Isorhamnetin-3-glucoside alleviates oxidative stress and opacification in selenite cataract in vitro. Toxicol In Vitro 24: $1662-1669,2010$.

4. Lee J, Jung E, Kim S, et al: Isorhamnetin represses adipogenesis in 3T3-L1 cells. Obesity (Silver Spring) 17: 226-232, 2009.

5. Jaramillo S, Lopez S, Varela LM, et al: The flavonol isorhamnetin exhibits cytotoxic effects on human colon cancer cells. J Agric Food Chem: Oct 5, 2010 (Epub ahead of print)

6. Kim JE, Lee DE, Lee KW, et al: Isorhamnetin suppresses skin cancer through direct inhibition of MEK1 and PI3-K. Cancer Prev Res (Phila) 4: 582-591, 2011.

7. Kong CS, Kim YA, Kim MM, et al: Flavonoid glycosides isolated from Salicornia herbacea inhibit matrix metalloproteinase in HT1080 cells. Toxicol In Vitro 22: 1742-1748, 2008.

8. Ma G, Yang C, Qu Y, Wei H, Zhang T and Zhang N: The flavonoid component isorhamnetin in vitro inhibits proliferation and induces apoptosis in Eca-109 cells. Chem Biol Interact 167: 153-160, 2007.

9. Teng BS, Lu YH, Wang ZT, Tao XY and Wei DZ: In vitro anti-tumor activity of isorhamnetin isolated from Hippophae rhamnoides L. against BEL-7402 cells. Pharmacol Res 54: 186-194, 2006.

10. Ferlay J, Shin HR, Bray F, Forman D, Mathers C and Parkin DM: Estimates of worldwide burden of cancer in 2008: GLOBOCAN 2008. Int J Cancer 127: 2893-2917, 2010.

11. Sartore-Bianchi A, Martini M, Molinari F, et al: PIK3CA mutations in colorectal cancer are associated with clinical resistance to EGFR-targeted monoclonal antibodies. Cancer Res 69: 1851-1857, 2009.

12. Samuels Y and Velculescu VE: Oncogenic mutations of PIK3CA in human cancers. Cell Cycle 3: 1221-1224, 2004.

13. Mosmann T: Rapid colorimetric assay for cellular growth and survival: application to proliferation and cytotoxicity assays. J Immunol Methods 65: 55-63, 1983.

14. Yang Y, Zhou X, Xiao M, et al: Discovery of chrysoeriol, a PI3K-AKT-mTOR pathway inhibitor with potent antitumor activity against human multiple myeloma cells in vitro. J Huazhong Univ Sci Technolog Med Sci 30: 734-740, 2010.
15. Jacinto E, Loewith R, Schmidt A, et al: Mammalian TOR complex 2 controls the actin cytoskeleton and is rapamycin insensitive. Nat Cell Biol 6: 1122-1128, 2004.

16. Sarbassov DD, Guertin DA, Ali SM and Sabatini DM: Phosphorylation and regulation of $\mathrm{Akt} / \mathrm{PKB}$ by the rictor-mTOR complex. Science 307: 1098-1101, 2005.

17. Morgensztern D and McLeod HL: PI3K/Akt/mTOR pathway as a target for cancer therapy. Anticancer Drugs 16: 797-803, 2005.

18. Yap TA, Garrett MD, Walton MI, Raynaud F, de Bono JS and Workman P: Targeting the PI3K-AKT-mTOR pathway: progress, pitfalls, and promises. Curr Opin Pharmacol 8: 393-412, 2008.

19. Tokunaga E, Oki E, Egashira A, et al: Deregulation of the Akt pathway in human cancer. Curr Cancer Drug Targets 8: 27-36, 2008.

20. Cheng JQ, Lindsley CW, Cheng GZ, Yang H and Nicosia SV: The Akt/PKB pathway: molecular target for cancer drug discovery. Oncogene 24: 7482-7492, 2005.

21. Hidalgo $\mathbf{M}$ and Rowinsky EK: The rapamycin-sensitive signal transduction pathway as a target for cancer therapy. Oncogene 19: 6680-6686, 2000.

22. Huang WY, Cai YZ and Zhang Y: Natural phenolic compounds from medicinal herbs and dietary plants: potential use for cancer prevention. Nutr Cancer 62: 1-20, 2010.

23. Jeong JH, An JY, Kwon YT, Rhee JG and Lee YJ: Effects of low dose quercetin: cancer cell-specific inhibition of cell cycle progression. J Cell Biochem 106: 73-82, 2009.

24. Shan BE, Wang MX and Li RQ: Quercetin inhibit human SW480 colon cancer growth in association with inhibition of cyclin D1 and survivin expression through $\mathrm{Wnt} / \beta$-catenin signaling pathway. Cancer Invest 27: 604-612, 2009.

25. Wang W, VanAlstyne PC, Irons KA, Chen S, Stewart JW and Birt DF: Individual and interactive effects of apigenin analogs on $\mathrm{G} 2 / \mathrm{M}$ cell-cycle arrest in human colon carcinoma cell lines. Nutr Cancer 48: 106-114, 2004.

26. Wan X, Harkavy B, Shen N, Grohar P and Helman LJ: Rapamycin induces feedback activation of Akt signaling through an IGF-1R-dependent mechanism. Oncogene 26: 1932-1940, 2007.

27. Hyun J, Shin SY, So KM, Lee YH and Lim Y: Isoflavones inhibit the clonogenicity of human colon cancer cells. Bioorg Med Chem Lett 22: 2664-2669, 2012

28. Abdul-Ghani R, Serra V, Gyorffy B, et al: The PI3K inhibitor LY294002 blocks drug export from resistant colon carcinoma cells overexpressing MRP1. Oncogene 25: 1743-1752, 2006.

29. Hoeffer CA and Klann E: mTOR signaling: at the crossroads of plasticity, memory and disease. Trends Neurosci 33: 67-75, 2010.

30. Bjornsti MA and Houghton PJ: The TOR pathway: a target for cancer therapy. Nat Rev Cancer 4: 335-348, 2004.

31. Nasmyth K: Viewpoint: putting the cell cycle in order. Science 274: 1643-1645, 1996.

32. Cappelletti V, Fioravanti L, Miodini P and Di Fronzo G: Genistein blocks breast cancer cells in the $\mathrm{G}(2) \mathrm{M}$ phase of the cell cycle. J Cell Biochem 79: 594-600, 2000. 